\title{
Muscle differentiation in blackspot seabream (Pagellus bogaraveo, Brunnich): Histochemical and immunohistochemical study of the fibre types
}

\author{
P. Silva ${ }^{\text {a,d }}$, A.M. Rowlerson ${ }^{\text {b }}$, L.M.P. Valente ${ }^{\mathrm{a}, \mathrm{d}}$, M. Olmedo $^{\mathrm{c}}$, \\ R.A.F. Monteiro ${ }^{\mathrm{a}, \mathrm{d}}$, E. Rocha ${ }^{\mathrm{a}, \mathrm{d}, *}$ \\ a Institute of Biomedical Sciences Abel Salazar - ICBAS, University of Porto, Portugal \\ b Applied Biomedical Research, School of Biomedical Sciences, King's College, University of London, United Kingdom \\ c Instituto Español de Oceanografia, Cabo Estay, Vigo, Spain \\ d Interdisciplinary Centre for Marine and Environmental Research - CIIMAR, Porto, Portugal \\ Received 24 November 2007; received in revised form 5 May 2008; accepted 22 May 2008 \\ Available online 14 July 2008
}

\begin{abstract}
The aim of this work was to gain insights into the mechanism of muscle differentiation and growth in Pagellus bogaraveo, by studying muscle fibre phenotypes identified by immunohistochemistry. At hatching, several layers of deep fast-white fibres were covered by a superficial fibre monolayer. At 5 days, slow-red fibres appeared near the lateral line nerve. At 40 days, the intermediate-pink muscle became visible, and in the slow-red and fast-white muscle layers transitions from larval myosin isoforms to the isoforms typical of adult muscle occurred. Between 70 and 100 days, small fibres with a distinct ATPase profile appeared throughout the fast-white muscle, marking the onset of "mosaic" hyperplasia. The myosin of the original superficial monolayer fibres underwent two myosin transformations, before being slowly replaced by an adult slow-red isoform. In juveniles and adults, the slow-red muscle layer could be resolved into two distinct types. The analysis of fibre phenotypes indicated that post-larval muscle growth occurred by two distinct stages of hyperplasia. This study offers a basis for further comparative and experimental studies with this economically relevant species, namely for identifying factors influencing its muscle growth dynamics and disclosing underlying mechanisms.
\end{abstract}

(C) 2008 Elsevier Ltd. All rights reserved.

Keywords: Pagellus bogaraveo; Fish; Differentiation; Muscle immunohistochemistry; Hyperplasia; Hypertrophy

\section{Introduction}

Skeletal muscle forms 40-60\% of the body mass in most fish. In contrast to mammalian muscle, which is characterised by a mixture of fibre types, the axial muscle of teleosts shows anatomically separate zones of muscle fibre types. The muscle fibres are separated in two layers: superficial slow-red muscle, with oxidative metabolism and slow contraction; and deep fast-white muscle, with glycolytic metabolism and fast contraction (Johnston et al., 1977; Bone, 1978). Between

\footnotetext{
* Corresponding author at: Lab. Histology and Embryology, Institute of Biomedical Sciences Abel Salazar - ICBAS, Lg. Prof. Abel Salazar, 24099 003 Porto, Portugal. Fax: +351 222062232.

E-mail address: erocha@icbas.up.pt (E. Rocha).
}

those layers, an intermediate muscle appears with fibres characterized as fast contracting with intermediate resistance to fatigue and intermediate speed of shortening (Johnston et al., 1977).

From pre-existing works it is clear that post-hatching muscle growth in fish involves both the enlargement of the already existing muscle fibres (hypertrophy) and the recruitment of new fibres (hyperplasia). During larval life, differentiation of slow-red and fast-white fibre types is supported by a hyperplastic mechanism that occurs by apposition of new fibres along proliferative zones, principally under the lateral line and in the apical myomere regions, and also just under the superficial monolayer (Rowlerson et al., 1995). The embryonic and larval phases of muscle growth are followed by a second period of myofibre formation in the fast-white 
muscle (Weatherley and Gill, 1981; Weatherley et al., 1988). The new wave of hyperplastic growth gives rise to a typical mosaic appearance of muscle fibres, as seen in transverse section, where fibres of different sizes intermingle (Carpenè and Veggetti, 1981; Rowlerson et al., 1985; Romanello et al., 1987; Scapolo et al., 1988). This second period of myofibre formation is greatly reduced or entirely absent in species that remain small in their ultimate size, such as Danio rerio (Hamilton) (Patterson et al., 2008) and Poecilia reticulata (Peters) (Veggetti et al., 1993), respectively. Thus, the early (embryonic and larval) 'stratified' hyperplasia occurs in all fish, but the extensive later (post-embryonic and most especially juvenile) 'mosaic' hyperplasia is namely a feature of the fish which reach large final sizes, and which are of most interest to commercial aquaculture (Rowlerson and Veggetti, 2001). In all fish, hypertrophic growth of the muscle fibres occurs at all life stages (until the maximum somatic size is reached), but it predominates in juveniles and adults (Veggetti et al., 1993; Rowlerson et al., 1995).

Myosin is a primary determinant of important contractile properties in vertebrate muscles, and exists in a variety of isoforms. In fish (as in other vertebrates) these can be distinguished by histochemical ATPase staining and by immunostaining with isoform-specific antimyosin antibodies-methods which can also be used to follow changes in isoform content occurring during development (e.g., as reviewed by, Carpenè and Veggetti, 1981; Scapolo et al., 1988; Crockford and Johnston, 1993; Veggetti et al., 1993; Johnston, 1994; Johnston and Horne, 1994; Johnston et al., 1998). Polyacrylamide electrophoresis and peptide mapping techniques can also be used to identify myosin isoforms, although these studies require the isolation of pure population of fibres and are mainly limited to fast muscle (Martinez et al., 1991; Focant et al., 1992; Johnston et al., 1998). Recently, new molecular techniques have been used, bringing also very important contributions to better understand fish muscle development and growth (e.g., as reviewed by, Chauvigné et al., 2006; Nihei et al., 2006; Steinbacher et al., 2006).

It is known that developmental transitions in myosin isoform expression are often linked to the formation of distinct 'waves' of fibres, which in fish are the initial embryonic, subsequent stratified and final mosaic phases of myogenesis (e.g., as reviewed by, Rowlerson and Veggetti, 2001; Sänger and Stoiber, 2001; Stellabotte and Devoto, 2007). The primary phase has been well studied in a very few species, most notably Danio rerio (Hamilton) (Devoto et al., 1996, 2006; Stickney et al., 2000), but the post-embryonic myogenic phases (stratified hyperplasia during larval life, and the later mosaic hyperplasia) have received much less attention. Larval growth is important, however, for survival in the first few weeks post-hatching, and the last phase, mosaic hyperplasia, is by far the greatest contributor to the muscle growth that brings fish to commercial size for aquaculture; so, these phases are of considerable practical as well as biological interest. We have, therefore, studied muscle development in
Pagellus bogaraveo (Brunnich), a fish under consideration as a potential candidate for Mediterranean aquaculture. In this study, we aimed to make a characterization of muscle differentiation and growth in this species, by focussing on: (1) the detailed aspects of the development of lateral muscle fibre phenotypes, especially their myosin isoforms, from hatching to juveniles and adult and (2) their correlation with the hyperplastic mechanisms of growth. Our approach employed both histochemical and immunohistochemical methods.

\section{Material and methods}

Larvae, fry and juveniles of Pagellus bogaraveo were obtained in the Instituto Español de Oceanografía (IEO) (Centro Oceanográfico de Vigo, España) and sexually mature adult fish from Mariculture Center of Calheta, in Madeira Island, Portugal. Spawning occurred from January to May (peaking in February-March) at $14 \pm 1{ }^{\circ} \mathrm{C}$, and embryonic incubation was performed at the same temperature. Embryonic life (fertilization to hatching) lasted only $54 \mathrm{~h}$. Larvae were maintained in the incubators at the natural temperature with running water until the day prior to mouth opening (at $115 \mathrm{~h}$ after hatching), at which they were transferred to the larvae culture tanks at $18 \pm 1{ }^{\circ} \mathrm{C}$. Fish feeding plan progressed from rotifers (fish age: 5-35 days), to Artemia naupli (fish age: $30-35$ days) and metanaupli (fish age: $35-45$ days), and finally to Gemma Micro diet (fish age: 45-60 days). Larvae aged from 0 to 23 days had 17 myotomes.

Fish were killed by anaesthetic overdose with MS222 (tricaine methane sulphonate). For histochemistry and immunohistochemistry whole fish were snap frozen in isopentane at $-80^{\circ} \mathrm{C}$. Before freezing, small individuals were combined with each other in composite blocks, being "sandwiched" with two slices of pig liver. Sections were cut at $10 \mu \mathrm{m}$, mounted on slides treated with 3aminopropyltriethoxysilane (Sigma Chemicals, Poole, UK) to improved section adhesion, and then stained histochemically for myosin ATPase and succinic dehydrogenase (SDH) activity or immunostained for myosin isoforms. Development of lateral muscle fibres and myosin isoforms was studied on transverse body sections and through a "cranial-caudal" examination as well as at different ages. Five antibodies specific for various isoforms of myosin were used: (1) 3-96: polyclonal anti-fish (Sparus aurata, Linnaeus) fast myosin; (2) 4-96: polyclonal anti-fish (Sparus aurata) slow myosin (Veggetti et al., 1999; Stoiber et al., 2002); (3) BA-G5: monoclonal anti-mammalian (Rattus norvegicus, Berkenhout) cardiac alpha myosin (Rudnicki et al., 1990); (4) SC-71: monoclonal anti-mammalian (Rattus norvegicus) type $2 \mathrm{~A}$ myosin (Schiaffino et al., 1989) and (5) BA-F8: monoclonal anti-mammalian (Rattus norvegicus) slow beta myosin (Borrione et al., 1988). The polyclonal antibodies were raised by AMR and colleagues, and the SC-71, BA-F8 and BAG5 monoclonals were obtained from hybridomas originally produced by Prof. S. Schiaffino and colleagues. 
A streptavidin-biotin-peroxidase immunohistochemistry kit was used (Histostain Plus; Zymed, San Francisco, CA), following the maker's instructions with minor adaptations. Firstly, endogenous avidin/biotin-binding activity was blocked and, after the slides were rinsed using a standardized washing scheme (used throughout) consisting of: $5 \mathrm{~min}$ in distilled water, $5 \mathrm{~min}$ (twice) in PBS, and $5 \mathrm{~min}$ in distilled water; $10 \%$ non-immune goat serum was applied for 120 min thereafter, followed by a long incubation step with the first antibody, at room temperature and for $48 \mathrm{~h}$, to ensure immunomarking through the full section thickness. After another wash in distilled water and in PBS, an incubation for 10 min with biotinylated secondary antibody followed. Then, sections were again washed and incubated for $10 \mathrm{~min}$ in a streptavidin-peroxidase solution. After rinsing in distilled water and PBS, the peroxidase activity was developed using $0.05 \% 3,3^{\prime}$-diaminobenzidine (DAB), in PBS and with $0.03 \% \mathrm{H}_{2} \mathrm{O}_{2}$, which gave a brown end product. Once rinsed in tap water, sections were mounted in DPX. Sections for which primary antibodies were omitted showed no immunomarking (negative controls).

Histochemistry of ATPase activity used a method described by Mascarello et al. (1986). In short, sections were pre-incubated for $5 \mathrm{~min}$ in $0.075 \mathrm{M} \mathrm{Na}$-barbital, $0.07 \mathrm{M}$ Na-acetate, $0.1 \mathrm{M} \mathrm{CaCl}_{2}$ buffer, adjusted with $\mathrm{NaOH}$, using a $\mathrm{pH}$ range from 9.8 to 10.6. After preincubation, sections were incubated in a $\mathrm{Na}_{2}$ ATP solution ( $1 \mathrm{mg} \mathrm{Na} 2 \mathrm{ATP} / \mathrm{ml}$ of the same buffer used for alkaline preincubation, adjusted to $\mathrm{pH} 9.4$ ) for $60 \mathrm{~min}$. Sections were mounted in DPX.

Histochemistry of SDH activity was revealed by incubation of sections in a solution of sodium succinate, at $\mathrm{pH} 7.4$ in $0.2 \mathrm{M}$ phosphate buffer. Nitroblue tetrazolium $(1 \mathrm{mg} / \mathrm{ml})$ was added to the solution prior to incubation, which was carried out in the dark until the stain developed (usually $120-180 \mathrm{~min})$. Sections were finally mounted in glycerol gelatine (Sigma).

\section{Results}

\subsection{Larvae aged 0 (hatching)-40 days}

At hatching (0 days), the myotomal muscle consisted of several layers of deep fibres and a monolayer of fibres that extended hypaxially and epaxially away from the lateral line. However, the most caudal (and therefore the most recently formed) myotome consisted of apparently only a complete monolayer, with no deep fibres. Deep fibres were polygonal in cross section and were bigger than those in the superficial monolayer. All fibres bound antibodies against both fast and slow fish myosins. The immunostaining was stronger in the superficial fibres than in the deep ones for all antibodies except BA-F8, which reacted weakly only with the deep fibres, showing that the fibres in those two muscle layers (LSM and LW) already differed in their myosin composition.
At hatching, the superficial monolayer fibres stained strongly with the myosin ATPase technique, following 5 min of alkaline pre-incubation at $\mathrm{pH} 9.8$, but no SDH activity could be demonstrated. The immunostaining patterns are shown in Table 1, and the time course of transitions is outlined in Fig. 1.

At 5 days (transition from endogenous to exogenous feeding) three layers of muscle fibres were present: superficial monolayer, slow-red (present only as one or two small diameter superficial fibres adjacent to the lateral line nerve, LR) (Fig. 1), and fast-white (forming the bulk of the muscle). These new slow-red fibres showed a particularly strong reaction with the monoclonal BA-G5 (Fig. 2A) and BAF8 antibodies and anti-fish slow myosin (Table 1). This immunostaining profile of LR fibres was similar to the later slow-red muscle (R2), although the ATPase activity was different (Table 1, Fig. 2C). These fibres were also SDH positive (Table 1; Fig. 2B).

The superficial monolayer fibres continued to stained strongly with the myosin ATPase technique; nonetheless, from 5 days showed higher SDH activity than in the deeper (fast-white) muscle layer (Figs. 2B and 3A). The same phenotypes were seen over the next few days (Fig. 3), although the number of fibres with the LR profile gradually increased in number (Fig. 3D).

At 23 days, the histochemical and immunohistochemical profiles of the superficial and deep fast-white fibres was unchanged except for a reduction in immunoreactivity of the superficial monolayer fibres with BA-G5 (Fig. 3D). In the deep muscle, there were no histochemical and immunohistochemical differences between the largest (most mature) and the smallest (presumed most recently formed) fibres. The muscle wall of the abdomen (derived from hypaxial myotomes) was formed by a small number of layers of fast fibres, externally covered with very small diameter fibres of the superficial monolayer type.

At 40 days, the superficial monolayer muscle now gave a positive reaction with BA-F8 (Table 1), suggesting a change to the isoform called juvenile superficial myosin (JSM1), as shown in Fig. 1. These fibres still had an ATPase activity stronger than that displayed by fast-white fibres.

At 40 days, all fibres of the deep layer were now immunonegative to 4-96 and BA-G5, suggesting a switch to a new isoform, called $\mathrm{W}$ in Table 1. At this age, a layer of slow-red fibres (with a strong SDH reaction) present at the lateral surface of the original superficial monolayer in a deeper position at the level of the horizontal septum showed a new staining profile (alkali-labile ATPase and immunopositive only with 4-96), attributed to a new slow myosin isoform (R1) in Table 1 . The immunoreactivity with 4-96 is shown in Fig. 4.

Also at 40 days, very small diameter fibres located at either side of the horizontal septum and just under the slow-red fibres were identified by their strongly anti-fish fast myosin (3-96) and anti-mammalian 2A myosin (SC-71) reactions 
Table 1

Summary of histochemical and immunohistochemical reactions of muscle fibre type in Pagellus bogaraveo

\begin{tabular}{|c|c|c|c|c|c|c|c|c|c|}
\hline \multirow[t]{2}{*}{ Muscle layer } & \multirow[t]{2}{*}{ Myosin isoform } & & \multicolumn{5}{|c|}{ Immunoreactivity for antibodies } & \multicolumn{2}{|c|}{ Histochemistry result } \\
\hline & & & $3-96$ & $4-96$ & SC-71 & BA-G5 & BA-F8 & ATPase & SDH \\
\hline \multirow{3}{*}{ Superficial monolayer (SM) } & LSM & - & ++ & ++ & ++ & ++ & - & ++ & ++ \\
\hline & JSM1 & - & ++ & + & ++ & + & + & ++ & ++ \\
\hline & JSM2 & - & - & + & - & + & + & ++ & ++ \\
\hline \multirow[t]{3}{*}{ Slow-red muscle (R) } & LR & - & - & +++ & - & +++ & +++ & - & ++ \\
\hline & $\mathrm{R} 1$ & - & - & +++ & - & - & - & - & ++ \\
\hline & $\mathrm{R} 2$ & - & - & +++ & - & +++ & ++ & ++ & ++ \\
\hline \multirow[t]{2}{*}{ Intermediate-pink muscle $(\mathrm{P})$} & $P$ & - & +++ & + & +++ & ++ & ++ & +++ & + \\
\hline & LW & - & + & + & + & + & + & + & - \\
\hline \multirow[t]{2}{*}{ Fast-white muscle (W) } & $\mathrm{W}$ & ld & ++ & - & + & - & + & + & - \\
\hline & & $\mathrm{sd}$ & ++ & - & + & - & + & ++ & - \\
\hline
\end{tabular}

LSM, JSM1 and JSM2, larval and juvenile superficial monolayer myosin isoforms; LR, R1 and R2, larval and adult slow-red myosin isoforms; LW and W, larval and adult fast-white myosin isoforms; $\mathrm{P}$, intermediate-pink myosin isoform; ld, large diameter fast-white fibres; sd, small diameter fast-white fibres; +++ strong positive reaction; ++ moderate positive reaction; + very weak reaction; - no reaction; 3-96 anti-fish fast myosin; 4-96: anti-fish slow myosin; BA-G5: anti-mammalian alpha-cardiac myosin; SC-71: anti-mammalian 2A myosin; BA-F8: anti-mammalian slow myosin.

(Table 1). Those fibres also had a very alkali-stable ATPase activity, and were therefore already "intermediate-pink" in type, with a myosin composition (here termed P) very similar, if not identical, to that found in juvenile and adult intermediate-pink muscle.

From 23 days, fibre phenotype profiles were the same for all myotomes studied, independent of their position along the cranio-caudal axis.

\subsection{Fry aged $70-100$ days}

By 70 days, the slow-red fibres had increased in number close to the horizontal septum, where they formed a layer several fibres deep. These fibres now also appeared epi- and hypaxially, as very small diameter fibres interspersed between the superficial monolayer fibres. The deep muscle showed a mosaic appearance, and a distinct interme-

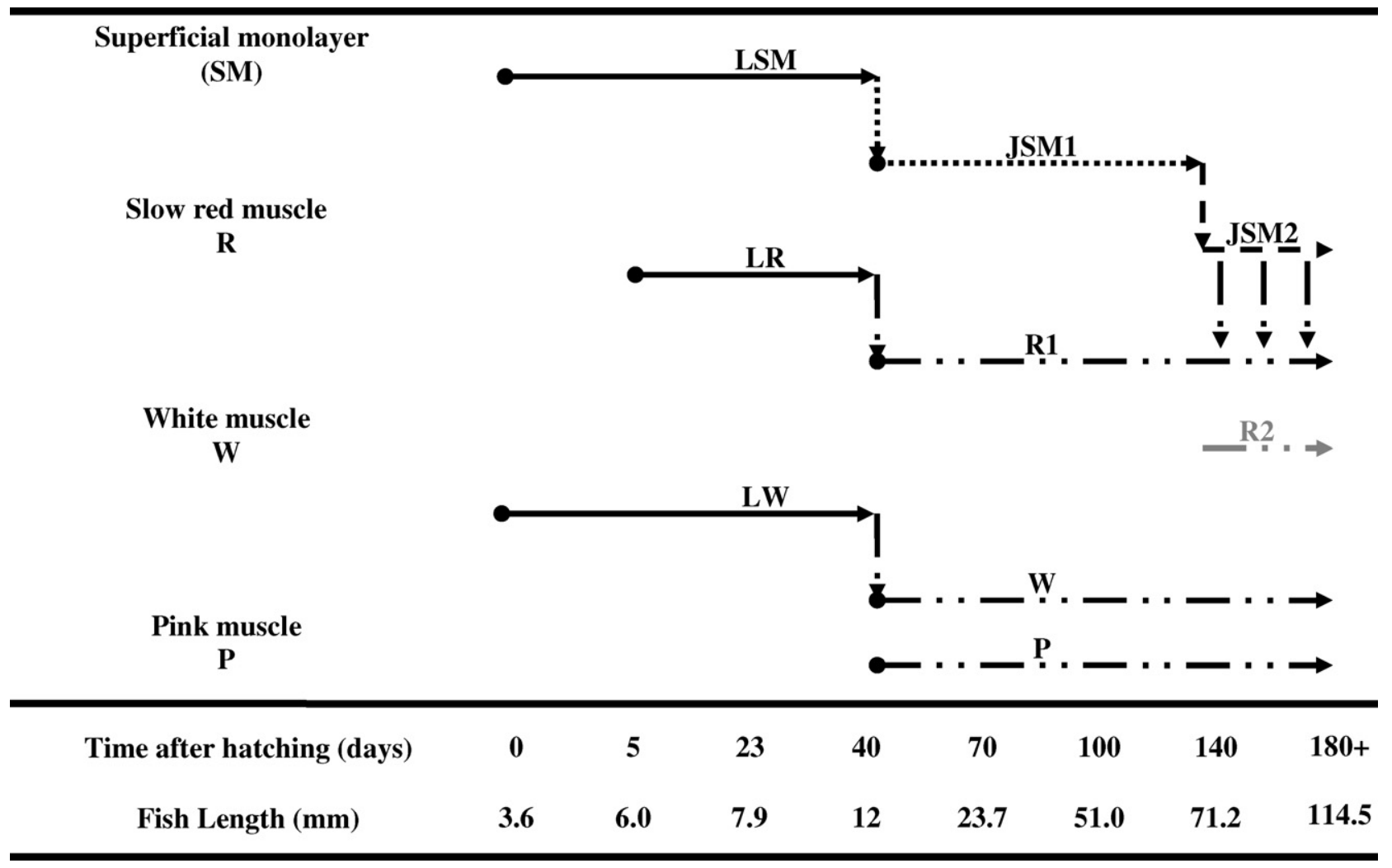

Fig. 1. Summary of myosin isoform transitions in the muscle layers during development. LSM, JSM1 and JSM2, larval and juvenile superficial monolayer myosin isoforms; LR, R1 and R2, larval and adult slow-red myosin isoforms; LW and W, larval and adult fast-white myosin isoforms; P, intermediate-pink myosin isoform. 


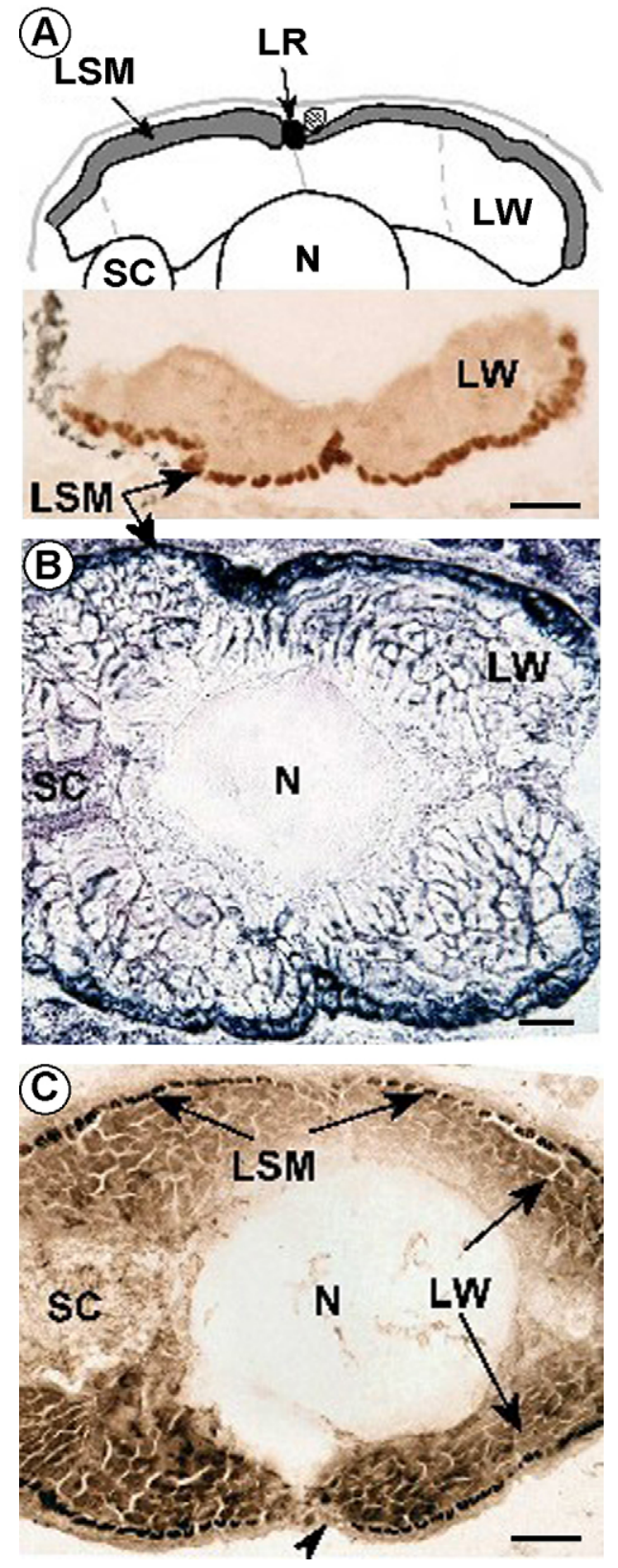

Fig. 2. Transverse sections of lateral muscle of Pagellus bogaraveo aged 5 days. (A) Immunostaining with BA-G5; (B) SDH; (C) alkali-stable mATPase. The superficial monolayer fibres (LSM) showed a strong immunoreaction with BA-G5 antibody and displayed strong ATPase and SDH activities. At this age, a small group of slow-red fibres (LR) appeared adjacent to the lateral line nerve- these are also strongly BA-G5 immunoreactive and SDH positive, but mATPase negative. Other symbols: SC, spinal cord, N, notochord, LW, fast-white muscle. Scale bar $=25 \mu \mathrm{m}$.

diate ('pink') zone inserted between slow-red and fast-white fibre domains was visible. Both deep and intermediate fibres reacted with anti-fish fast myosin (3-96); nevertheless, reaction was stronger in the latter zone. The ATPase technique showed that the fast-muscle layer had a mosaic appearance in all individuals examined (Fig. 5). The small diameter fibres stained stronger than the adjacent larger ones even though they had the same immunostaining profile.
At 100 days, small diameter fibres with the ATPase and immunostaining profile of intermediate-pink muscle appeared in a limited deep area of the fast-muscle, at either side of the dorso-ventral septum, in both the epaxial and hypaxial quadrants. In parallel, the slow-red muscle layer became thicker not only near the horizontal septum but also hypo- and epiaxially. Slow-red fibres were also present along the horizontal septum, near the notochord. At that age, the immunohistochemical and histochemical reactions remained unchanged.

\subsection{Juveniles aged from 140 days to adult}

In juveniles, significant changes occurred in the slow-red muscle layers. These showed changes in fibre type properties, and also grew in thickness by addition of more fibres, especially at the level of the horizontal septum, giving the typical wedge shape also seen in adult fish.

At 140 days, the superficial muscle was multi-layered close to the horizontal septum and as its fibres were now anti-fish fast myosin (3-96) and anti-mammalian 2A myosin (SC-71) negative, the myosin immunostaining profile present in those fibres is called JSM2 (Table 1; Fig. 1). Between 140 and 180 days, the myosin immunoreactivity of superficial fibres underwent a third transition, as the JSM2 profile was replaced by a slow myosin isoform (R1), which had both the immunoreactivity and histochemical ATPase and SDH activities typical of red (slow) muscle (Table 1). At 180 days, the superficial fibres with JSM2 myosin profile were present only in the superficial muscle zones located farthest from the horizontal septum.

Surprisingly, and at the end of juvenile life, the slowred muscle at the level of horizontal septum next to the intermediate-pink muscle, could be resolved into two distinct types by immunostaining against mammalian myosin antibodies (Fig. 6) and also by ATPase activity: 1) an innermost layer of fibres with a myosin profile (R2) similar to the latest isoform (JSM2) found in the superficial monolayer fibres (Table 1); and 2) an exterior layer of fibres displaying the usual adult slow myosin profile (R1). There were no changes in the immunostaining or histochemical profile of the intermediate-pink fibres. In adult fish, the number of fibres with the R2 profile increased sufficiently to form a layer several fibres deep separating the main slow-red muscle (R1, i.e., BA-G5 and BA-F8 negative) from the underlying intermediate-pink muscle (characterized by the SC-71 immunoreactivity). The "pink" muscle showed a mosaic pattern of intermediate-pink and fast-white fibre types (Fig. 6A, $\mathrm{C}$ and $\mathrm{E})$.

\section{Discussion}

The aim of our study was to follow post-embryonic developmental changes in myosin composition and other fibre type properties (such as oxidative activity) in lateral muscle of 

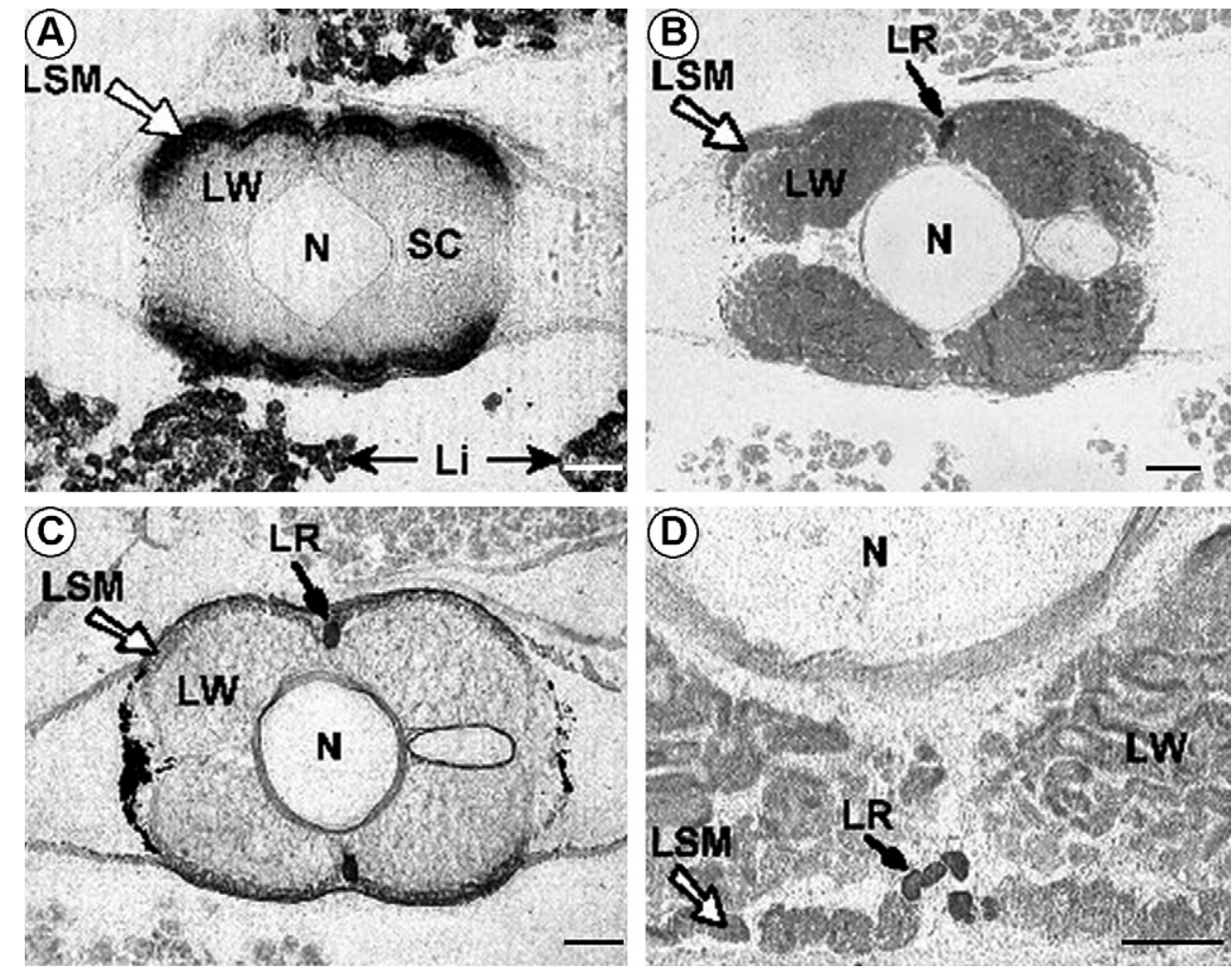

Fig. 3. Transverse sections of lateral muscle of Pagellus bogaraveo aged 11 (A-C) and 23 (D) days. (A) SDH; (B and D) immunoreaction with BA-G5; (C): immunoreaction with 4-96. The superficial monolayer fibres (LSM) stain strongly for SDH, and weakly with BA-G5, whereas the new slow-red fibres (LR) close to the lateral line nerve are very strongly stained with both 4-96 and BA-G5. Fast-white (LW) muscle fibres are negative for all reactions. Li indicates liver, used as support tissue when the larvae were snap-frozen. Scale bar $A, B$, and $C=50 \mu \mathrm{m}$ and $D=25 \mu \mathrm{m}$. Other symbols: SC, spinal cord, $\mathrm{N}$, notochord.

Pagellus bogaraveo, relating those changes to the appearance of new fibres from hatching to adult life. We used a panel of isoform-specific antibodies for identifying developmental transitions in myosin expression, as well as histochemical staining for myosin ATPase and for SDH enzyme activity (a marker for mitochondrial content and oxidative metabolism). Some of the antibodies were monoclonals raised against mammalian myosins the structural homology of which with fish myosins is not known, so immunoreactivity with those antibodies is therefore used only to distinguish between myosins (to give a reactivity profile), rather than for identifying them as a specific type. However, other antibodies were raised against myosins from fish and, therefore, can be used for identifying myosins in Pagellus bogaraveo as 'fast' or 'slow'. The last antibodies were examined by the Western Blotting method and the main reaction was with the myosin heavy chain. All the evidence presented in this paper was based not only on the antibodies reaction but also on ATPase staining. Together, they allowed us to characterize several different isoform profiles appearing at specific locations and developmental stages, as discussed below.

\subsection{Larvae aged 0 (hatching)-40 days}

At hatching, lateral muscle in Pagellus bogaraveo consisted of several layers of deep fast-white fibres covered by a typical superficial fibre monolayer. The latter extended into the edges of the hypo- and epaxial regions and represented the only muscle layer of the most caudal myomeres, as observed in Sparus aurata by Mascarello et al. (1995). At hatching, the superficial monolayer was already differentiated from the deep muscle with regard to its myosin composition (compare LSM and LW profiles in Table 1), although neither resembled the adult types. The existence of both fibre types is in accordance with the finding that the first phase muscle formation occurs quite early in most teleosts and is, in fact, a sequential process in itself, beginning with adaxial ("primary slow fibre") myogenesis and continuing with fast fibre myogenesis (Devoto et al., 1996; Stickney et al., 2000; Steinbacher et al., 2006). Several studies in Danio rerio showed that initial muscle formation is comprised of at least two different myogenic cell types (Devoto et al., 1996; Blagden et al., 1997). Provided that co-expression of two or more isoforms cannot be excluded (and transient expression of a 'fast' myosin in superficial fibres has been described in trout (Oncorhynchus mykiss, Walbaum) larvae by Chauvigné et al., 2006), the overall staining profile of the SM myosin does not fit what could be expected from a simple mix; so it is more likely due to the presence of a specific isoform. Again, this resembles the situation seen in Sparus aurata (Mascarello et al., 1995).

At 5 days of age (transition from endogenous to exogenous feeding), a third muscle fibre type profile (LR) appeared in 

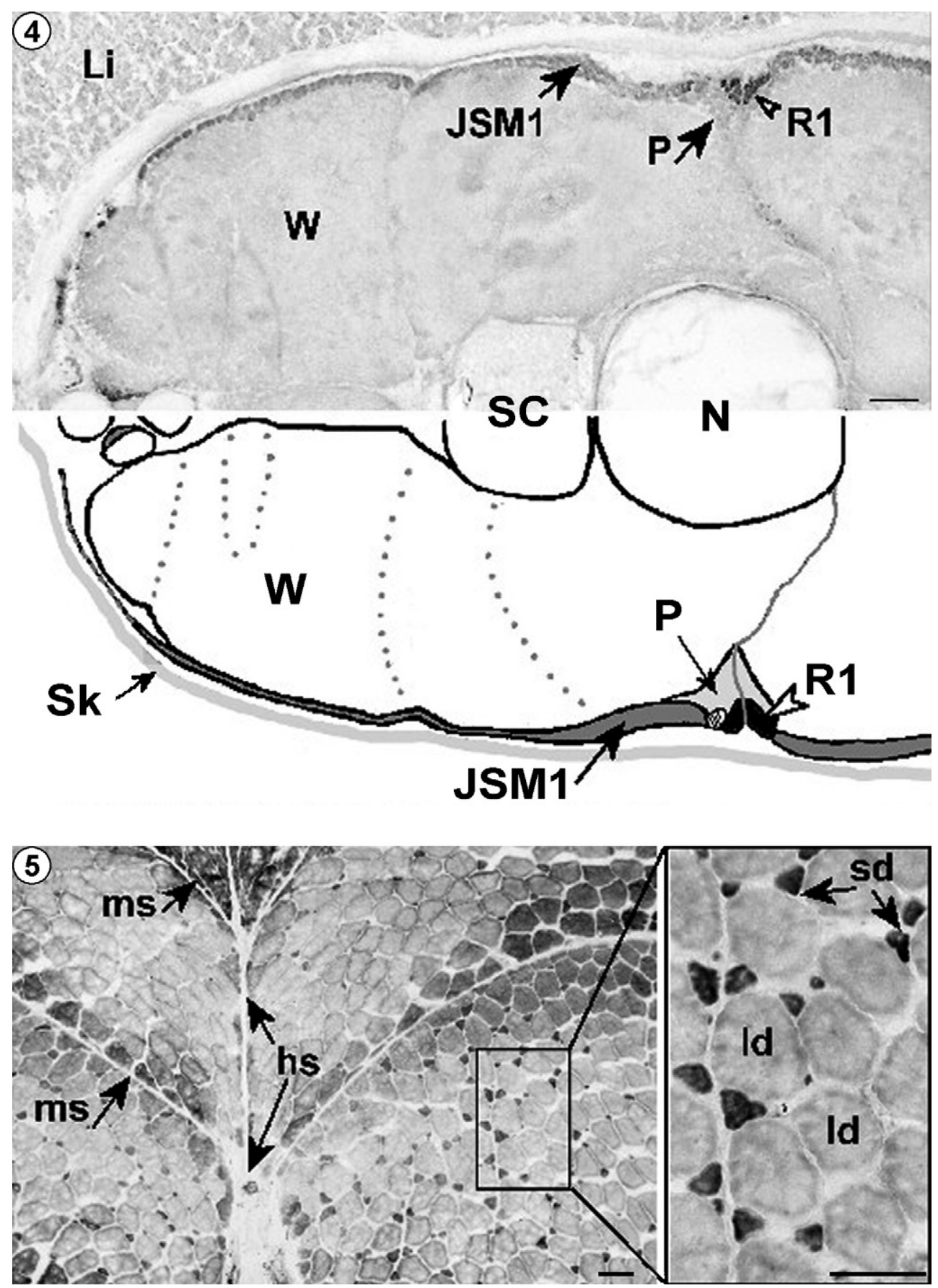

Figs. 4-5. Fig. 4 shows transverse sections of one epaxial quadrant of lateral muscle of Pagellus bogaraveo aged 40 days, immunoreaction with 4-96. Fast-white muscle (W) is immunonegative, most superficial monolayer fibres now have the JSM1 profile (including weak staining with 4-96), and the number of slow-red fibres (now with R1 myosin profile, and strongly reactive with 4-96) has increased. Other symbols: Sk, skin; SC, spinal cord; N, notochord; P, intermediate-pink muscle; Li, liver (suppport tissue), continuous grey line running from R1 to notochord, horizontal septum, dotted grey lines, position of myosepts. Scale bar $=50 \mu \mathrm{m}$. Fig. 5 shows transverse section of lateral muscle (fast-white portion) of Pagellus bogaraveo aged 70 days, stained for mATPase activity after mild alkalai pre-incubation. This shows the "mosaic appearance" of the fast-white muscle. Boxed area is shown at higher magnification on the right. Symbols: ld, large diameter fibres; sd, small diameter fibres; ms, myosept; hs, horizontal septum. Scale bar $=50 \mu \mathrm{m}$.

a small group of fibres adjacent to the lateral line nerve, and differed from one of the definitive slow-red muscle isoforms only in its ATPase staining (Table 1—compare LR with R2). Like the superficial monolayer fibres, those were positive for $\mathrm{SDH}$, and the combination of their position and overall stain- ing profile suggests that these are the first fibres of the adult slow-red muscle layer. A molecular characterisation of cell recruitment over the three phases of myogenesis was carried out in Rutilus meidingeri (Heckel) (Steinbacher et al., 2006) and it was found that the zones where expression of 



Fig. 6. Transverse sections in the epaxial region of lateral muscle of Pagellus bogaraveo aged 180 days. (A and B) Serial sections immunostained with 4-96 and BA-F8, respectively; for orientation, the same two fibres are indicated with arrows. There is a small amount of intermingling of the intermediate-pink (4-96 weakly positive, BA-F8 positive) and slow-red (4-96 positive, BA-F8 negative) fibres at the junction between these layers. (C, D and E) Serial sections from an adjacent area, immunostained for BA-G5 (C), BA-F8 (D) and SC-71 (E). This shows the junction between intermediate-pink and slow-red muscle at higher power. Intermediate-pink fibres $(\mathrm{P})$ are strongly SC71-immunoreactive, whereas the slow-red fibres have 2 different immunoreactivity profiles: R1, indicated by the open triangles (BA-G5 and BA-F8 negative) or R2, indicated by the filled circles (BA-G5 and BA-F8 positive). Scale bar $A$ and $B=100 \mu \mathrm{m}$ and $C, D$ and $E=50 \mu \mathrm{m}$.

muscle-specific transcriptional activators arise at the lateral of the slow fibre monolayer next to the horizontal septum were entirely slow type, which is an accordance with our hypothesis. To the best of our knowledge, however, the cellular origin of the newly larval slow-red fibres it is not clearly identified. Most recent work, reviewed by Stellabotte and Devoto (2007), provides a possible explanation of the original source of at least some of the myogenic cells involved in teleost muscle hyperplasia. They suggested that as in the amniotes, such cells could originate from the dermomyotome, which have now been proved to also exist in fish in the form of the socalled external cell layer. An external cell origin of the new slow muscle fibres emerging from the lateral surface next to the lateral line nerve was supposed by several authors in different fish species as Danio rerio (Waterman, 1969), Clupea harengus (Linnaeus) (Johnston, 1993), Dicentrarchus labrax (Linnaeus) and Sparus aurata (Linnaeus) (Veggetti et al., 1990; López-Albors et al., 1998). As seen in other fish (Scapolo et al., 1988; Mascarello et al., 1995; Patruno et al., 1998), the appearance of those fibres coincided with the first attempts of the larvae at cruise swimming in search for food, and may be an important factor in larval survival at that age.
At the end of larval life (23-40 days), all fibres of the deep layer lost their LW reaction profile and acquired the fast-white profile (W) typical of large diameter fast-white muscle in adult fish. A similar transition from one myosin isoform to another in the deep muscle was also observed in Dicentrarchus labrax (Linneaus) (Scapolo et al., 1988), but it differs from the situation found in Sparus aurata, another Sparid species (Mascarello et al., 1995) in which no change could be detected.

Information about developmental myosins in fish is currently very uneven. For example, peptide mapping studies in charr (Salvelinus alpinus, Linnaeus) by Martinez et al. (1991) and in herring (Clupea harengus, Linnaeus) by Johnston et al. (1998) have revealed developmental changes in myosin heavy chain composition in fast-white muscle, whereas in Brycon moorei (Steindachner), the same technique showed only minor differences in the chemical composition of myosin heavy chain expressed at various larval and juvenile stages (Huriaux et al., 2003). Molecular biology techniques such as those employed by Chauvigné et al. (2006) and Nihei et al. (2006), for following the expression of isoforms at mRNA level may eventually prove more informative in this regard. 
At 40 days of larval life (weaning, i.e., transition from live to formula food), the superficial monolayer fibres acquired a new immunoreactivity profile (JSM1), and the increasing number of new slow-red fibres located just under the superficial monolayer around the lateral line lost their reaction with BA-G5 and BA-F8 monoclonal antibodies, thereby acquiring the typical profile of myosin in adult slow-red muscle (a transition from LR to R1, see Table 1).

Also at 40 days, an additional fibre type was identified, forming a distinctive 'pink' or intermediate layer. The combination of very alkali-stable ATPase, strong reaction with 3/96 (specific for fish fast myosin) and an intermediate staining for SDH is typical of intermediate-pink fibres in many (although not all) fish species (e.g., as reviewed by, Mascarello et al., 1986, 1995; Sänger and Stoiber, 2001). The strong reaction observed in the intermediate-pink fibres with the antibody for fish fast myosin is in accordance with the recent results obtained in the Steinbacher et al. (2006) study. Using molecular techniques it was found that the new muscle cells arising at the lateral growth zones of Rutilus meidingeri are fast type rather than intermediate. It was suggested that the myosin heavy chain (MyHC) expressed by intermediate fibres diverged only slightly from fast fibre isoforms in their nucleotide sequence, thus causing a crossreaction of the fast MyHC. According to Johnston (1980), the intermediate-pink fibres can be considered functionally analogous to the oxidative fast twitch fibres of other vertebrates, and contribute to the cruise swimming at intermediate speeds.

Thus, in Pagellus bogaraveo the three main fibre types seen in later juvenile and adult life had appeared by the end of larval life, in positions within the myotome typical of the stratified hyperplastic growth phase. This pattern was observed in another Sparid fish, Sparus aurata (Mascarello et al., 1995), but it is different from the situation observed in another large, fast growing fish, Dicentrarchus labrax, in which the large diameter fast fibres do not acquire their definitive mATPase profile until a later life stage (Scapolo et al., 1988). The significance of this species variation is as yet unknown.

\subsection{Fry aged $70-100$ days}

The mosaic appearance of fast-white muscle is the result of the third hyperplastic process and generally occurs only in fish, which grow to a large final size (Carpenè and Veggetti, 1981; Rowlerson and Veggetti, 2001; Sänger and Stoiber, 2001; Johnston, 2006). In Pagellus bogaraveo small diameter fibres appeared in the fast-white muscle between 70 and 100 days post-hatching, i.e., in early juvenile life, and this was reflected in a wide range of fibre sizes that gave the mosaic appearance in cross sections. The ATPase and immunoreactivity profiles of the small diameter fibres in mosaic fast-white muscle vary widely among species (Rowlerson et al., 1985; Romanello et al., 1987; Scapolo et al., 1988; Higgins, 1990;
Sänger and Stoiber, 2001). However, in Pagellus bogaraveo we observed the same picture found in Sparus aurata (Mascarello et al., 1995), where the small diameter fibres in the fast-white muscle differed from the large diameter ones only in their ATPase reactivity. With this study we do not know if mosaic hyperplasia started after the previous phase had ceased or overlapped with stratified growth. The examination of the MyoD and Myogenin (members of the myogenic regulatory family) expression in Salmo trutta lacustris (Linnaeus) showed that the stratified hyperplasia and mosaic hyperplasia began simultaneously and progressed vigorously (Steinbacher et al., 2007). A similar approach can be used in future in Pagellus bogaraveo to clarify this issue. To the best of our knowledge, the origin of the myogenic cells involved in mosaic hyperplasia in teleosts remains unknown. Recent research using lineage analysis techniques suggested that either dermomyotome cells or a dermomyotome-independent population of cells could be responsible for mosaic hyperplasia (Stellabotte and Devoto, 2007).

Mosaic hyperplasia is quantitatively the most important phase of myogenesis (Johnston, 2006), and also allows for the more effective and faster locomotion, which is important for juveniles. The intensity of the mosaic hyperplastic process gradually wanes in older fish, although in some fish it may also be seasonal (Carpenè and Veggetti, 1981; Alami-Durante et al., 2007). The very interesting study by Alami-Durante et al. (2007) also shows that juvenile hyperplastic muscle growth is affected by earlier thermal life history, an effect presumably operating at the level of the myogenic cell population(s), which is the substrate for the mosaic growth phase.

Between 40 and 180 days, the myosin composition of superficial muscle fibres underwent a further transition, as the superficial monolayer isoforms JSM1 was gradually replaced by the JSM2 profile summarized in Table 1 . As the original superficial monolayer fibres are eventually subsumed into the adult slow-red muscle layer, it is likely that the JSM2 profile does not represent a distinct isoform, but rather the near-complete transition to the R2 isoform of the slow-red muscle layer. Thus, monolayer fibres underwent three transformations from their original composition to the slow-red type. This transformation began in all monolayer fibres close to the lateral line, and gradually proceeded epi- and hypoaxially.

The definitive slow-red muscle was formed gradually due to the combined mechanism of hyperplastic growth forming new slow-red fibres with additional conversion of all the monolayer fibres into slow-red muscle fibres, a phenomenon already described in other species (Scapolo et al., 1988; Veggetti et al., 1993; Mascarello et al., 1995; Patruno et al., 1998).

\subsection{Juveniles aged from 140 days to adult}

At these ages, the axial muscle consisted mainly of fastwhite fibres covered by a intermediate-pink layer and above that a slow-red layer. Unexpectedly, the slow-red layer could 
be resolved into two distinct types by myosin immunostaining: one (R1) with the expected slow-red myosin isoform (alkali-labile ATPase and a strong reaction with polyclonal antibody 4/96), and the other (R2) which had a positive immunoreactivity with two of the monoclonal antibodies as well as with 4/96, had an alkali-stable ATPase activity, and was present in fibres closest to the underlying intermediatepink muscle. Heterogeneity of the slow-red muscle layer in fish is not unknown, but none of the kinds previously described-slow-red muscle rim fibres (van Raamsdonk et al., 1980), tonic fibres (Chayen et al., 1993; Sänger and Stoiber, 2001), and the ATPase mosaic due to hyperplastic growth in Sparus aurata (Mascarello et al., 1995), are equivalent. The Ictalurus melas (Rafiesque) has been described as having two distinct layers of intermediate-pink muscle as shown by ATPase staining (Mascarello et al., 1986), but as the outer one of these had an alkali-labile ATPase, it is presumably not equivalent to the R2 profile in Pagellus bogaraveo. Also in Dicentrarchus labrax juveniles heterogeneity in the superficial muscle was observed, due to the appearance of small groups of very small diameter fibres with a rosette-like appearance which have more alkali- and acid-stable mATPase activity (Scapolo et al., 1988). In Pagellus bogaraveo we obtained a similar ATPase result, although, the slow-red fibres did not have the "rosette" arrangement observed in Dicentrarchus labrax. Moreover, the antibodies used in this study were completely different from the ones used in the Dicentrarchus labrax study, so no direct comparison between the fibres immuno-profile could be done. The pattern of expression of myosins in relation to the development stage and muscle type is complex. Nihei et al. (2006) isolated six myosin heavy chains (MYH) DNAs from carp (Cyprinus carpio, Linnaeus) and revealed that MYHs predominantly associated with slow muscle or early development stages were expressed in the fast muscle of adult fish at some acclimation temperatures but not others. Our study does not allow conclusions about the exact nature of the R2 myosin composition; although the combination of differences in both ATPase activity and immunoreactivity do suggest they contain different isoforms of myosin, we cannot at present exclude the possibility that those are hybrid fibres expressing both the JSM2 and R1 forms. We hope to investigate this in future, by other means.

Apart from some obvious anatomical differences, the arrangement of the three main fibre types was shared by all the myomeres of the trunk. Caudally, the deep muscle layer tended to become gradually thinner; in contrast, the slow-red muscle layer continued to be well developed both epi- and hypaxially. This was also seen in the majority of species where it was observed that slow-red muscle occurs as a continuous mid-lateral strip, the relative dimension of which increases in rostro-caudal direction, achieving maximal values in the posterior part of the body (Bone, 1978). The finding that most of the red muscle is in the posterior part of Pagellus bogaraveo further supports the idea that most power for steady swimming at moderate speeds comes from posterior rather than anterior musculature (Luther et al., 1995). As Pagellus bogaraveo is a benthopelagic fish (Bauchot and Hureau, 1990), this result also suggests that the possession of a relatively high proportion of slow-red muscle is not confined to active, pelagic fish (Luther et al., 1995). In general, the rostral myomeres showed more advanced and organized developmental stages than the caudal ones at early ages, following the rostro-caudal myogenesis rule of most fish (Johnston et al., 1996).

\section{Conclusions}

From the histochemistry and immunoreactivity of fibres in lateral muscle of Pagellus bogaraveo, various developmental sequences of myosin isoform transitions were identified over the period from hatching to adult. Superficial monolayer fibres appeared to transform from their original larval composition firstly into two intermediate forms (at 40 days and 140 days of age) and then gradually to the slow-red type. The pink (intermediate) muscle layer was the last major layer to appear, at about weaning. The deep fast-white muscle initially grew by stratified hyperplasia, but shortly after weaning (fry stage, $c a$. 70 days) mosaic hyperplasia was detected. The axial muscle of juveniles, thus consisted mainly of fast-white fibres, covered by thin layers of intermediate-pink and slowred muscle fibres. The slow-red fibres could be divided in to two distinct subtypes by differential myosin immunostaining and ATPase activity, neither of which corresponded to slowred muscle rim or tonic fibres. This was an unexpected and unprecedented finding, the significance of which is not yet apparent.

Overall, this study showed that changes in the fibre phenotype reflected the developmental events in lateral muscle of Pagellus bogaraveo, and although many features resembled events in Sparus aurata, there were also differences (such as the two subtypes of slow-red muscle in the older fish). Information about developmental transitions in myosin isoform expression in fish muscle is still very fragmentary the methods used are indeed different, and too few species have been examined throughout development in order we may have a clear picture of what are common features and what are species-specific variations that may be related to the particular environmental conditions or life-style. However, our study does provide additional support for the notion that developmental transitions in myosin isoform expression in muscle do occur in fish as in mammals, and are linked, at least in part, to the different phases of fibre formation. In this study, in which fish were raised in conditions of water salinity and temperature close to those found in the natural environment, we have established the basic timeline of events for Pagellus bogaraveo. This is crucial for supporting future comparative and experimental studies targeting muscle growth dynamics and its underlying mechanisms in a species, which has considerable potential for commercial aquaculture. 


\section{Acknowledgements}

Work partially supported by FCT PhD Grant SFRHBD-14068-2003 attributed to P. Silva and FCT pluriannual projects attributed to R.A.F. Monteiro and E. Rocha. The authors are greatly indebted to the "Instituto Español de Oceanografía" (Centro Oceanográfico de Vigo, España) and Mariculture Centre of Calheta (Madeira Island, Portugal), which provided the fish. We thank Prof. Dr. Rui Henrique, Head of the "Serviço de Anatomia Patológica" of the IPOPorto for access to facilities. Thanks also to the British Council (Treaty of Windsor Programme) for a travel grant (to LMPV and AMR) to support this collaboration.

\section{References}

Alami-Durante, H., Olive, N., Rouel, M., 2007. Early thermal history significantly affects the seasonal hyperplastic process occurring in the myotomal white muscle of Dicentrarchus labrax juveniles. Cell Tissue Res. 327, 553-570.

Bauchot, M.-L., Hureau, J.C., 1990. Sparidae. In: Quero, J.C., Hureau, J.C., Karrer, C., Post, A., Saldanha, L. (Eds.), Check-List of the Fishes of the Eastern Tropical Atlantic (CLOFETA), vol. 2. JNICT/SEI, Lisbon, Paris, UNESCO, pp. 790-812.

Blagden, C.S., Currie, P.D., Ingham, P.W., Hughes, S.M., 1997. Notochord induction of zebrafish slow muscle mediated by Sonic hedgehog. Genes Dev. 11, 2163-2175.

Bone, Q., 1978. Locomotor muscle. In: Hoar, W.S., Randall, D.J. (Eds.), Fish Physiology, vol. VII. Academic Press.

Borrione, A.C., Zanellato, A.M.C., Saggin, L., Mazzoli, M., Azzarello, G., Sartore, S., 1988. Neonatal myosin heavy chains are not expressed in Ni-induced rat rhabdomyosarcoma. Differentiation 38, 49-59.

Carpenè, E., Veggetti, A., 1981. Increase in muscle fibres in the lateralis muscle (white portion) of Mugilidae (Pisces, Teleostei). Experientia 37, 191-193.

Chauvigné, F., Ralliere, C., Cauty, C., Rescan, P.Y., 2006. In situ hybridisation of a large repertoire of muscle-specific transcripts in fish larvae: the new superficial slow-twitch fibres exhibit characteristics of fast-twitch differentiation. J. Exp. Biol. 209, 372-379.

Chayen, N.E., Rowlerson, A.M., Squire, J.M., 1993. Fish muscle structure: fibre types in flatfish and mullet fin muscles using histochemistry and myosin antibody labelling. J. Mus. Res. Cell Motil. 14, 533542.

Crockford, T., Johnston, I.A., 1993. Development changes in the composition of myofibrillar proteins in the swimming muscles of Atlantic herring. Clupea harengus. Mar. Biol. 115, 15-22.

Devoto, S.H., Melancon, E., Eisen, J.S., Westerfield, M., 1996. Identification of separate slow and fast muscle precursor cells in vivo, prior to somite formation. Development 122, 3371-3380.

Devoto, S.H., Stoiber, W., Hammond, C.L., Steinbacher, P., Haslett, J.R., Barresi, M.J.F., Patterson, S.E., Adiarte, E.G., Hughes, S.M., 2006. Generality of vertebrate developmental patterns: evidence for a dermomyotome in fish. Evol. Dev. 8 (1), 101-110.

Focant, B., Huriaux, F., Vandewalle, P., Castelli, M., Goessens, G., 1992. Myosin, parvalbumin and myofibril expression in barbell (Barbus barbus L.) lateral white muscle during development. Fish Physiol. Biochem. 10, 133-143.

Higgins, P.J., 1990. The histochemistry of muscle in juvenile Atlantic salmon, Salmo salar L. J. Fish Biol. 37, 521-529.

Huriaux, F., Baras, E., Vandewalle, P., Focant, B., 2003. Expression of myofibrillar proteins and parvalbumin isoforms in white muscle of dorada during development. J. Fish Biol. 62, 774-792.
Johnston, I.A., 1980. Specialization of fish muscle. In: Goldspink, D.F. (Ed.), Development and Specialization of Skeletal Muscle. Cambridge University Press, Cambridge, London, New York, pp. 123-148.

Johnston, I.A., 1993. Temperature influences muscle differentiation and the relative timing of organogenesis in herring (Clupea harengus) larvae. Mar. Biol. 116, 363-379.

Johnston, I.A., 1994. Development and plasticity of fish muscle with growth. Basic Appl. Myol. 4, 353-368.

Johnston, I.A., 2006. Environment and plasticity of myogenesis in teleost fish. J. Exp. Biol. 209, 2249-2264.

Johnston, I.A., Horne, Z., 1994. Immunocytochemical investigations of muscle differentiation in the Atlantic herring (Clupea harengus: teleostei). J. Mar. Biol. Assoc. U.K. 74, 79-91.

Johnston, I.A., Davison, W., Goldspink, G., 1977. Energy metabolism of carp swimming muscles. J. Comp. Physiol. 114, 203-216.

Johnston, I.A., Vieira, V.L.A., Hill, J., Temperature and ontogeny in ectotherms: muscle phenotype in fish 1996. In: Johnston, I.A., Bennett, A.F. (Eds.), Phenotypic and Evolutionary Adaptation of Organisms to Temperature. Society of Experimental Biology, Seminar Series. Cambridge University Press, pp. 153-181.

Johnston, I.A., Cole, N.J., Abercromby, M., Vieira, V.L.A., 1998. Embryonic temperature modulates muscle growth characteristics in larval and juvenile herring. J. Exp. Biol. 201, 623-646.

López-Albors, O., Gil, L., Ramírez-Zarzoza, G., Vázquez, J.M., Latorre, R., Garcia-Alcácar, A., Arencibia, A., Moreno, F., 1998. Muscle development in gilthead sea bream (Sparus aurata, L.) and sea bass (Dicentrarchus labrax, L.): further histochemical and ultrastructural aspects. Anat. Histol. Embryol. 27, 223-229.

Luther, P.K., Munro, P.M.G., Squire, J.M., 1995. Muscle ultrastructure in the teleost fish. Micron 26, 431-459.

Martinez, I., Christiansen, J.S., Ofstad, R., Olsen, R.L., 1991. Comparison of myosin isoenzymes present in skeletal and cardiac muscles of the Arctic charr Salvelinus alpinus (L.): sequential expression of different myosin heavy chains during development of the fast white muscle. Eur. J. Biochem. 195, 743-753.

Mascarello, F., Romanello, M.G., Scapolo, P.A., 1986. Histochemical and immunohistochemical profile of pink muscle fibres in some teleosts. Histochemistry 84, 251-255.

Mascarello, F., Rowlerson, A., Radaelli, P., Veggetti, A., 1995. Differentiation and growth of muscle in the fish Sparus aurata (L.): I. Myosin expression and organization of fibre types in lateral muscle from hatching to adult. J. Muscle Res. Cell Motil. 16, 213-222.

Nihei, Y., Kobiyamal, A., Ikeda, D., Ono, Y., Ohara, S., Cole, N.J., Johnston, I.A., Watabe, S., 2006. Molecular cloning and mRNA expression analysis of carp embryonic, slow and cardiac myosin heavy chain isoforms. J. Exp. Biol. 209, 188-198.

Patruno, M., Radaelli, G., Mascarello, F., Carnevali, M.D.C., 1998. Muscle growth in response to changing demands of functions in the teleost Sparus aurata (L.) during development from hatching to juvenile. Anat. Embryol. 198, 487-504.

Patterson, S.E., Mook, L.B., Devoto, S.H., 2008. Growth in the larval zebrafish pectoral fin and trunk musculature. Dev. Dynam. 237, 307-315.

Romanello, M.G., Scapolo, P.A., Luprano, S., Mascarello, F., 1987. Postlarval growth in the lateral white muscle of the eel, Anguilla anguilla. J. Fish Biol. 30, 161-172.

Rowlerson, A., Veggetti, A., 2001. Cellular mechanisms of post-embryonic muscle growth in aquaculture species. In: Johnston, I.A. (Ed.), Muscle Development and Growth. Fish Physiology series, vol. 18. Academic Press, San Diego, pp. 103-140.

Rowlerson, A., Scapolo, P.A., Mascarello, F., Carpenè, E., Veggetti, A., 1985. Comparative study of myosin present in lateral muscle of some fish: species variations in myosin isoforms and their distribution in red, pink and white muscle. J. Muscle Res. Cell Motil. 6, 601-640.

Rowlerson, A., Mascarello, F., Radaelli, G., Veggetti, A., 1995. Differentiation and growth of muscle in the fish Sparus aurata (L.): II. Hyperplastic and hypertrophic growth of lateral muscle from hatching to adult. J. Muscle Res. Cell Motil. 16, 223-236. 
Rudnicki, M.A., Jackowski, G., Saggin, L., McBurney, M.W., 1990. Actin and myosin expression during development of cardiac muscle from cultured embryonal carcinoma cells. Dev. Biol. 138, 348-358.

Sänger, A.M., Stoiber, W., 2001. Muscle fiber diversity and plasticity. In: Johnston, I.A. (Ed.), Muscle Development and Growth. Fish Physiology Series, vol. 18. Academic Press, San Diego, pp. 187-250.

Scapolo, P.A., Veggetti, A., Mascarello, F., Romanello, M.G., 1988. Development transitions of myosin isoforms and organisation of the lateral muscle in the teleost Dicentrarchus labrax (L.). Anat. Embryol. 178, 287-295.

Schiaffino, S., Gorza, L., Sartore, S., Sggin, L., Ausoni, S., Vianello, M., Gundersen, K., Lømo, T., 1989. Three myosin heavy chain isoforms in type 2 skeletal muscle fibres. J. Mus. Res. Cell Motil. 10, 197-205.

Steinbacher, P., Haslett, J.R., Six, M., Gollmann, H.P., Sänger, A.M., Stoiber, W., 2006. Phases of myogenic cell activation and possible role of dermomyotome cells in teleost muscle formation. Dev. Dynam. 235, 3132-3143.

Steinbacher, P., Haslett, A., Obermayer, A., Marschallinger, H.C., Bauer, H.C., Sänger, A.M., Stoiber, W., 2007. MyoD and Myogenin expression during myogenic phases in brown trout: a precocious onset of mosaic hyperplasia is a prerequisite for fast somatic growth. Dev. Dynamic. 236, 1106-1114.

Stellabotte, F., Devoto, S.H., 2007. The teleost dermomyotome. Dev. Dynam. 236, 2432-2443.

Stickney, H.L., Barresi, M.J.F., Devoto, S.H., 2000. Somite development in zebrafish. Dev. Dynam. 219, 287-303.
Stoiber, W., Haslett, J.R., Wenk, R., Steinbacher, P., Gollmann, H.P., Sänger, A.M., 2002. Cellularity changes in developing red and white fish muscle at different temperatures: simulating natural environmental conditions for a temperature freshwater cyprinid. J. Exp. Biol. 205, 2349-2364.

van Raamsdonk, W., te Kronnie, G., Pool, C.W., van Laarse, W., 1980. An immune histochemical and enzyme characterization of the muscle fibres in myotomal muscle of the teleost Brachydanio rerio, HamiltonBuchanan. Acta Histochem. 67, 200-216.

Veggetti, A., Mascarello, F., Scapolo, P.A., Rowlerson, A., 1990. Hyperplastic and hypertrophic growth of lateral muscle in Dicentrarchus labrax (L.). An ultraestrutral and morphometric study. Anat Embryol 182, 1-10.

Veggetti, A., Mascarello, F., Scapolo, P.A., Rowlerson, A., Carnevali, C.M.D., 1993. Muscle growth and myosin isoform transitions during development of a small teleost fish, Poecilia reticulata (Peters) (Atheriniformes, Poeciliidae): a histochemical, immunohistochemical, ultrastructural and morphometric study. Anat. Embryol. 187, 353-361.

Veggetti, A., Rowlerson, A., Radaelli, G., Arrighi, S., Domeneghini, C., 1999. Post-hatching development of the gut and lateral muscle in the sole. J. Fish Biol. 55 (Suppl. A), 44-65.

Waterman, R.E., 1969. Development of the lateral musculature in the teleost, Brachydanio rerio: a fine structural study. Am. J. Anat. 125, 457-494.

Weatherley, A.H., Gill, H.S., 1981. Characteristics of mosaic muscle growth in rainbow trout Salmo gairdneri. Experientia 37, 1102-1103.

Weatherley, A.H., Gill, H.S., Lobo, A.F., 1988. Recruitment and maximal diameter of axial muscle fibres in teleosts and their relationship to somatic growth and ultimate size. J. Fish Biol. 33, 851-859. 